\title{
Numerical Study of Heat Transfer in an Air-to-Air Heat Exchanger Integrated with a Thermoelectric Heat Pump
}

\author{
Seong-Yong Cheon ${ }^{1}$, Hye-Won Dong ${ }^{1}$, Yong-Kwon Kang ${ }^{1}$, Soo-Jin Lee ${ }^{1}$, Jae-Weon Jeong \\ ${ }^{1}$ Department of Architectural Engineering, Hanyang University, Seoul, Republic of Korea
}

\begin{abstract}
The main objective of this study was to propose a thermoelectric heat-pump unit design for integration with an air-to-air heat exchanger as an air-condenser or evaporator, and to estimate the heat exchanger effectiveness and outlet air temperature based on numerical analyses. Accordingly, a numerical model was investigated to estimate the effectiveness of the crossflow heat exchanger. The heating and cooling sources of the thermoelectric module (TEM) were in direct contact with the airstream from the thermoelectric heat pump (TEHP) unit. Flat-plate fin types were used, which are commonly used in simple heat exchangers to increase the heat transfer area. The numerical model was developed in MATLAB to estimate the heat transfer effectiveness and the air temperature of the outlet side. Numerical analyses were conducted with the proposed model in twodimensions. The numerical simulation results were compared with actual data obtained from a prototype TEHP unit to validate the proposed model. The results showed that the outlet air temperature and fin effectiveness were associated with $20 \%$ error bounds. This numerical simulation model can be used as a tool for the consideration of the heat transfer effectiveness and outlet temperatures in the design of heat exchangers that are attached on the thermoelectric module.
\end{abstract}

\section{Introduction}

Vapor compression technologies have recently received tremendous attention as heating and cooling source devices in HVAC systems. The vapor compression system has a high-coefficient of performance (COP) and can be integrated with the HVAC system. However, there is a limitation in that it uses artificial refrigerant that has negative impacts on the environment (Creazzo et al., 2015). Therefore, nonvapor compression technologies can be directly applicable to HVAC systems, and the thermoelectric module (TEM) is one of the devices that can be used as alternatives to the vapor compression system (Rowe, 2005; Lee et al., 2015). Simulation and other studies have been previously conducted in which the effectiveness of the integration of the TEM with the HVAC system had been assessed (Lim and Jeong, 2018; $\mathrm{Kim}$ et al, 2017). However, most of the conducted simulation studies were either simplified or were conducted based on assumed heat transfer profiles between the source and the fluids that are critical to the TEM performance.
The main objective of this study was a) to propose a design for a thermoelectric heat-pump unit for its integration with a heat sink for use as an air condenser or evaporator, and b) to estimate the heat exchanger effectiveness based on numerical analyses. In this study, a numerical model was developed to estimate the effectiveness of the cross-flow heat exchanger type. The heating and cooling sources of the thermoelectric module (TEM) were in direct contact with the airstream in the TEHP unit. Flat plate fin types were used to increase the heat transfer area. These are commonly used in simple heat exchangers. To estimate the heat transfer and outlet air temperature in this unit, a two-dimensional numerical model was developed in MATLAB. To validate the proposed model, the simulation results were compared with actual data obtained from a prototype TEHP unit. This numerical simulation model can serve as a testing tool for the evaluation of the heat transfer effectiveness and for designing the heat exchanger attached on the thermoelectric module.

\section{System and simulation model}

The target simulation model is the thermoelectric heat pump unit (TEHP unit) that is used to achieve heating and cooling to the air stream, as shown in Figure 1. The numerical model was developed to estimate the heat exchange effectiveness and the outlet air temperature at each stream side. The results of the model were validated by comparison with experimental data.

\section{Air-to-air thermoelectric heat pump unit}

Given that the thermoelectric module (TEM) is a solid heat pump system, it acts as a heating and cooling source simultaneously and is supplied by electric power. The TEHP unit consists of TEMs and a heat sink to transfer the heat from the TEM source to the air stream.

The proposed unit is an air-to-air TEHP unit, as shown in Figure 1. The TEHP unit has two air flow channels which are constructed in such a way that the air flow channels (i.e., the primary and secondary air flows) are not mixed with each other. The TEHP unit has two operation modes: a cooling priority mode and a heating priority mode. In the cooling priority mode of the TEHP, the primary air is cooled by the cooling source of the TEM, while the secondary air is heated by the rejected heat of the TEM. When TEHP operates according to the heating priority mode, the primary air is heated by changing the electric current direction into the TEMs. In this case, it would not be necessary to supply a secondary air flow - that has 
relatively low-air temperature, e.g., during the winter season - to maintain the heating condition. In this study, a simulation model was developed to predict the cooling priority mode of the TEHP unit.

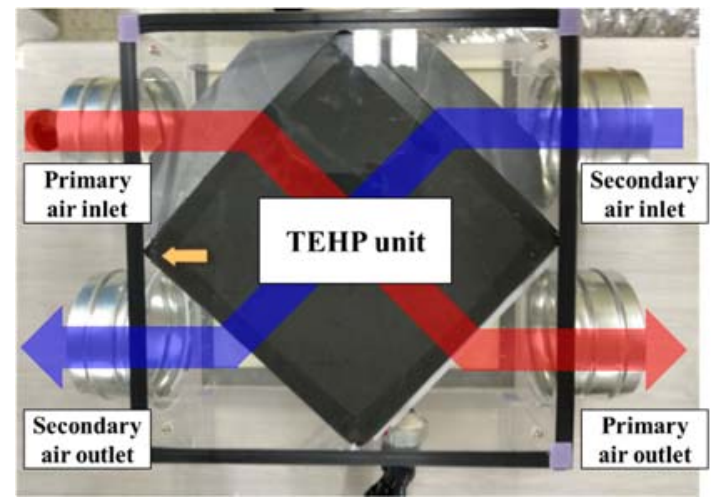

Figure 1: Configuration of air-to-air thermoelectric heat pump unit.

To validate the simulations with the actual experiments, the simulated heat pump unit was designed according to the dimensions of a prototype heat pump unit, as shown in Figure 2. The TEHP unit consisted of primary and secondary air channels, TEM layers, and insulation. The air channel was a flat-plate fin channel, and was constructed with aluminum. The TEM layer was inserted between the primary and secondary air channels as a sandwich structure. The size of the TEHP had a width and length equal to $298 \mathrm{~mm}$, and a height equal to $172 \mathrm{~mm}$. The TEM layer contained nine TEMs, which were arranged within a rectangular grid. The insulation was attached in such a way to prevent the unwanted heat transfer, such as the heat interference of the TEMs and the surrounding space.

To estimate the performance of the TEHP unit, we measured various parameters: inlet and outlet air temperatures, the surface temperatures of the TEMs, and the air flow rate. The thermal performance of the TEHP was defined by the heat exchange effectiveness between each source of the TEM and the air stream temperature. In this simulation, the condensation, which is affected by thermal performance of the TEHP unit, was not considered. The TEHP unit was only operated to supply sensible cooling and heating to the air stream.



a. Thermoelectric heat pump unit

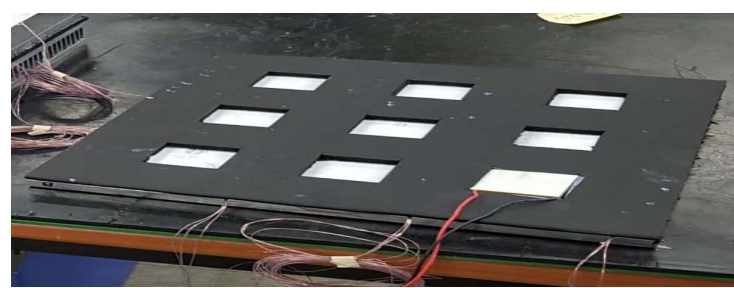

b. Thermoelectric module layer

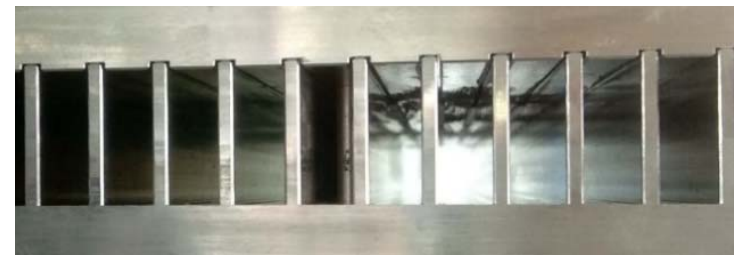

c. Air-flow channel

Figure 2: Detail schematics of prototype thermoelectric heat pump unit,

\section{Model of the thermoelectric heat pump system}

Figure 3 shows a schematic of the numerical model. The TEHP unit is a cross-flow type. The nodes of the simulation models distinguish the air streams. In other words, nodes are associated with areas that directly face the TEM (TEM nodes), while insulation nodes are associated with the area that is affected by the air stream heat exchange process. In the TEM nodes, the primary air is cooled by the cooling source of the TEM, and the secondary air is heated by the heating source of the TEM. In this simulation, it was assumed that heat fluxes on the cooling and heating sources of the TEMs were constant. In the insulation nodes, the heat transfer was only affected by the primary and secondary air temperatures and the air flow rate. The simulation input value was defined based on the primary and secondary air temperatures, air flow rate, and the electric current that affected the cooling and heating source capacity. The simulation model calculated each parameter, including the surface temperature of the hot and cold sides of the TEMs, the average outlet air temperature, and the heat exchanger effectiveness of each side.

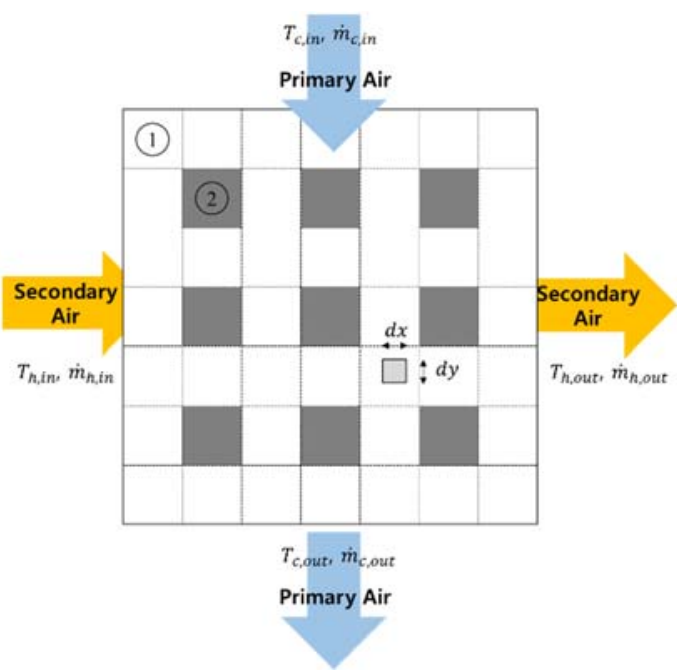

a. Floor diagram of the TEHP unit 


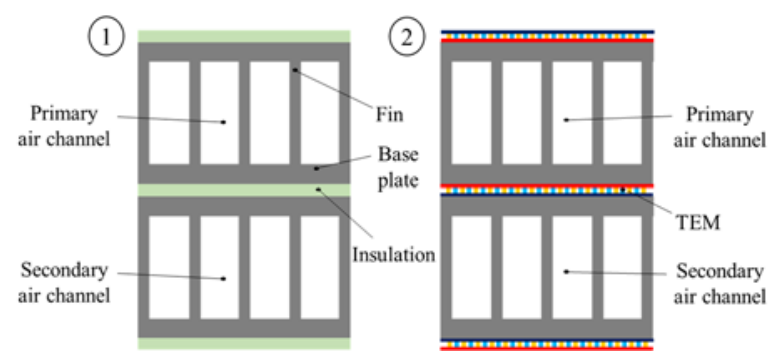

b. Section diagram of the TEHP unit

Figure 3: Numerical model of thermoelectric heat pump.

Figure 4 shows the heat transfer diagram with the thermal resistances in the TEM and insulation nodes. In the TEM nodes, the heat flows from the hot side of the TEM to the air stream in the heated part. By contrast, in the cooling part, the heat flows from the air stream to the cold side of the TEM. The heat resistance between the air stream and the surface temperature of the TEM is defined according to the heat sink of the fins and the conduction heat of the base plate, as formulated by Equations (1)-(4). The thermal resistance of the heat sink is determined by the number, conduction, thickness, and the distance between the fins, and the velocity of the fluid. In the insulation nodes, the heat flow is attributed to air temperature difference of the hot and cold sides of the heat sink. The total heat resistance is dependent on the hot and cold sides of the fin, the conduction heat transfer of the base plate, and the insulation. a. TEM node

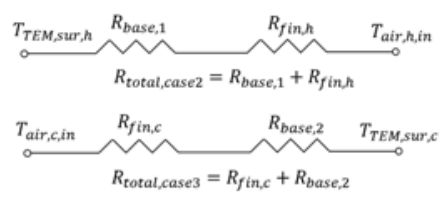

b. Insulation node $T_{\text {air }, h, i n}^{R_{\text {fin, h }}}$
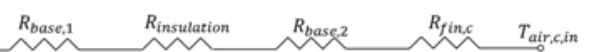

Figure 4: Heat transfer diagram and thermal resistances.

$$
\begin{gathered}
R_{\text {total }, \text { fin }}=\frac{\Delta T_{\text {max }}}{\mathrm{N}_{\text {fin }} \phi_{i} \eta_{\text {fin }}} \\
\eta_{\text {fin }}=\frac{\tanh (X)}{X}, \quad X=\sqrt{N u_{m . t}^{\infty} Y}, \quad Y=\frac{k_{f} l_{\text {fin }}^{2}}{k_{s} D_{\text {fin }} t_{f i n}} \\
N u_{m . t}^{\infty}=\frac{D_{h, f i n}}{k_{f}} \frac{\phi_{i}}{2 L_{\text {channel }} l_{\text {fin }} \Delta T_{\text {max }}} \\
\phi_{i}=\rho_{\text {air }} c_{p, \text { air }} l_{\text {fin }} D_{\text {fin }} U_{\text {air }} \Delta T_{\text {max }}\left[1-e^{\left(-4 L^{*} N u_{m, T}\right)}\right]
\end{gathered}
$$

In the TEM node, it is necessary to estimate the heat capacity at the hot and cold sides. The heat capacity of TEM was estimated using Equations (5) and (6), which consisted of terms associated with the Seebeck, Joule, and Fourier effects. To estimate the heat capacities of the cooling and heating sides of the TEM, the temperatures of all the surfaces of the TEM should be defined. The heat capacities of the hot and cold sides of the TEM are balanced by the heat transferred from the air to the surface according to the thermal resistance, as defined by Equations (7) and (8). To achieve the referred balance in TEM with air, the calculation is iterated such that the errors-expressed as the percentage difference between the heat capacity of the theoretical TEM and the heat transfer to the air side - are lower than $0.1 \%$ by adjusting the temperature value of each surface. The heat capacities of the TEM on the hot and cold sides were calculated at the end of the loop. The flow diagrammatic representation of the estimation of the heat capacity of the TEM is shown in Figure 5.

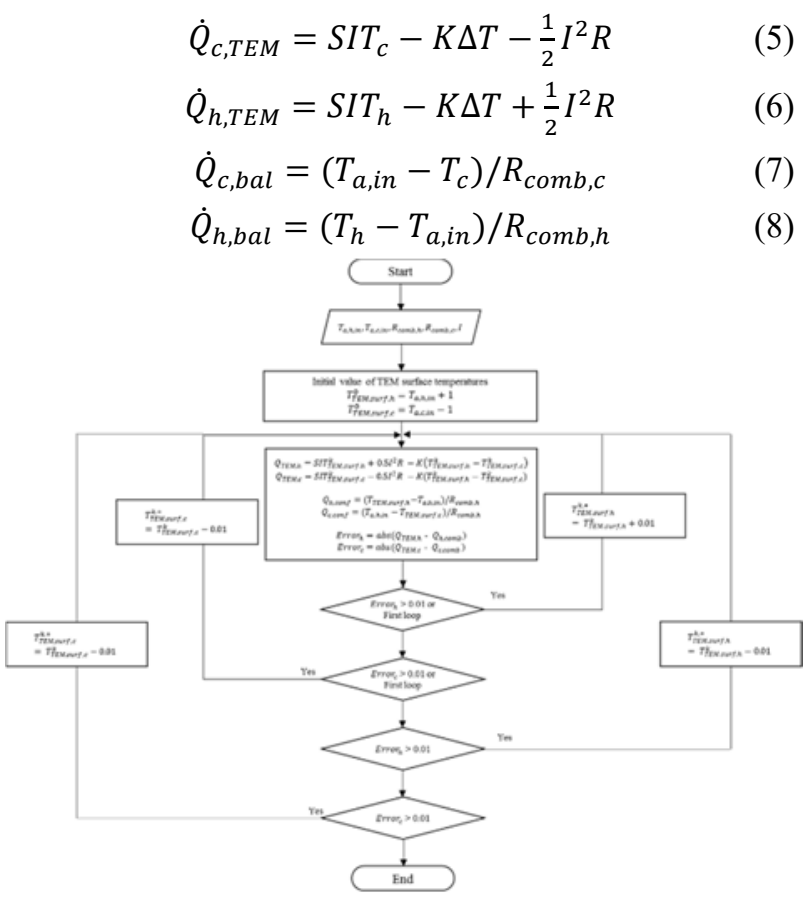

Figure 5: Simulation logic of the thermoelectric module.

\section{Simulation results and discussion}

The performance of the TEHP unit was quantified based on the estimates from numerical simulations and actual measurements of each outlet air temperature and each surface temperature, as shown in Figure 6. One set of the simulation results showed that the hot side surface temperature of the TEM was $55.8^{\circ} \mathrm{C}$, with $58^{\circ} \mathrm{C}$ as the upper and $52^{\circ} \mathrm{C}$ as the lower values of the TEHP unit. Additionally, the surface temperature of the TEM at the hot side was shown to be $56.5^{\circ} \mathrm{C}$ according to numerical simulations. The surface temperature of the TEM on the cold side was $4^{\circ} \mathrm{C}$, with $4.3^{\circ} \mathrm{C}$ as the upper and $3.1^{\circ} \mathrm{C}$ as the lower values, while the surface temperature of the cold side was $3.8^{\circ} \mathrm{C}$ according to simulations. The outlet air temperature of the hot side was measured and was found to range from $30.1^{\circ} \mathrm{C}$ to $33^{\circ} \mathrm{C}$ (average value of $32^{\circ} \mathrm{C}$ ). Meanwhile, the numerical simulation result of the air temperature at the hot side was $33.9^{\circ} \mathrm{C}$. Finally, the outlet air temperature of the cold side was measured and was found to range from $7.1^{\circ} \mathrm{C}$ to $8^{\circ} \mathrm{C}$ (average value of $7.3^{\circ} \mathrm{C}$ ). Furthermore, the numerical simulation results yielded an air temperature of $6.8^{\circ} \mathrm{C}$ for the cold side.

The simulated temperature results were slightly higher for the hot side and lower for the cold side. This is attributed to the influence of the heat loss on ambient air despite the use of insulation in the effort to prevent the unwanted heat exchange. 


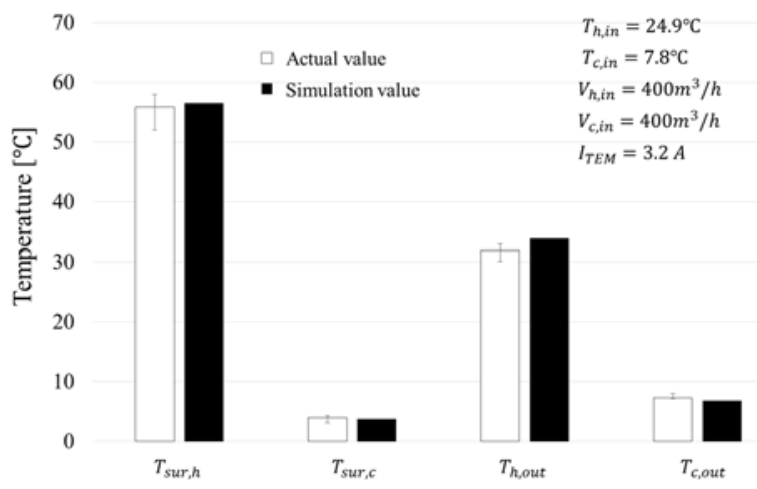

Figure 6: Temperature variance between actual measurements and simulations.

The heat exchange effectiveness was formulated to generalize the heat transfer effect at the hot and cold sides to represent the performance of the TEHP unit, as shown by Equations (9) and (10). The heat exchange effectiveness values (hot and cold sides) of the TEHP unit were bound by $20 \%$ error margins compared to the actual values. The heat exchange effectiveness was directly affected by the two main design factors, i.e., the thermal resistance between the source of the TEM and the air stream, and the heat transfer area associated with the TEM.

$$
\begin{gathered}
\varepsilon_{h}=\left(T_{\text {sur }, h}-T_{h, \text { out }}\right) /\left(T_{\text {sur }, h}-T_{h, \text { in }}\right) \\
\varepsilon_{c}=\left(T_{c, \text { out }}-T_{\text {sur }, c}\right) /\left(T_{c, \text { in }}-T_{\text {sur }, c}\right)
\end{gathered}
$$

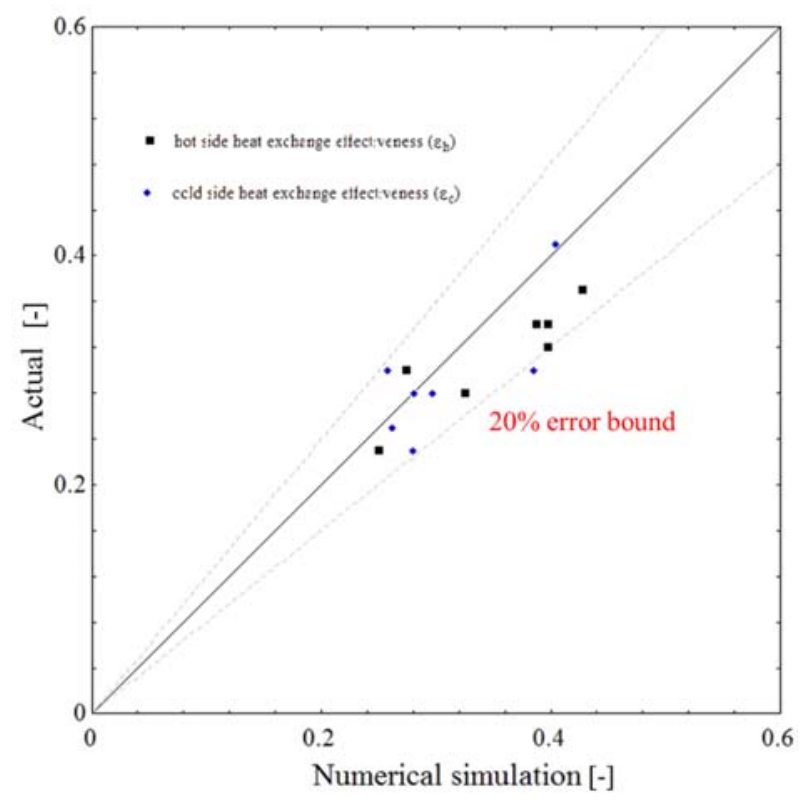

Figure 7: Heat exchange effectiveness based on actual measurements and numerical simulations.

The performance of the TEHP unit was estimated according to the effect of the overall thermal resistance and the facing area ratio. The overall thermal resistance is the thermal resistance of the combined heat transfer of the heat sink with the air stream. The facing area ratio is the ratio of the TEMs surface area and the total plan area.

Each component value is plotted in Figure 8 by considering the variation of the overall thermal resistance from $0.05 \mathrm{~K} / \mathrm{W}$ to $0.5 \mathrm{~K} / \mathrm{W}$. The decrease of the thermal resistance between the source and the air stream led to an increase in the heat exchange effectiveness from 0.29 to 0.54 . The results showed that the surface temperature difference between the hot and cold sides of the TEM decreased from $52^{\circ} \mathrm{C}$ to $40^{\circ} \mathrm{C}$. This means that the heat capacity of the TEM was dependent by the air stream. However, the limitation imposed by the increasing heat exchanger effectiveness was manifested by the bypassed air, and was not directly affected by the heating and cooling capacity of the TEMs.

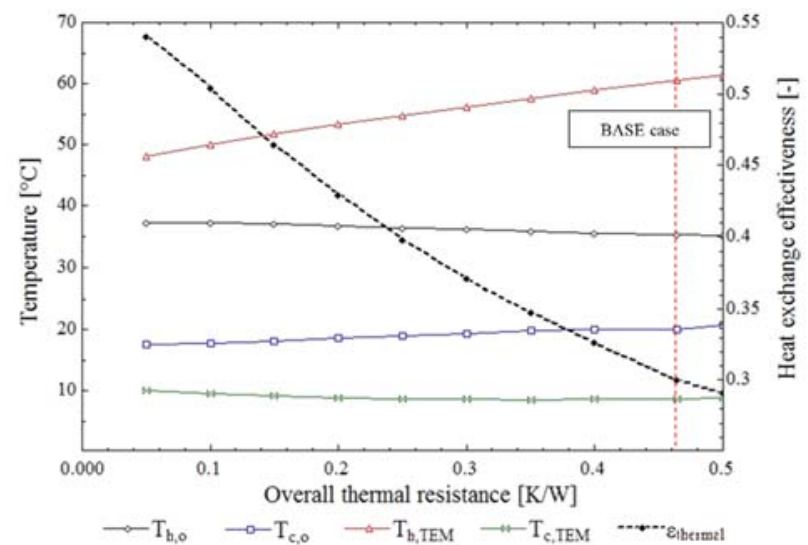

Figure 8: Overall thermal resistance of the TEHP unit

In Figure 9, each component value was plotted by considering a variation of the facing area ratio in the range from 0.56 to 1.5 . The increase of the facing area ratio led to the increase of the heat exchange effectiveness from 0.29 to 0.38 in $0.46 \mathrm{~K} / \mathrm{W}$ of the overall thermal resistance. The value of the heat exchange effectiveness increased as a function of the facing area ratio of the TEM. There was a limit to the increase of the exchange effectiveness that depended on the adjustment of the facing area ratio. Although the air stream was directly connected by the heating and cooling source of the TEM, the increase of the heat exchange effectiveness was limited because the heat transfer from the source of the TEM to the air stream was not sufficient.

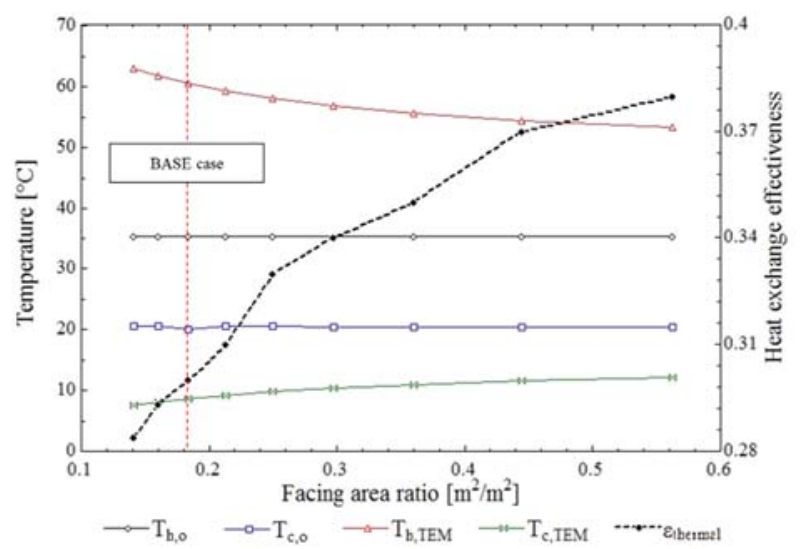

Figure 9: Facing area ratio of the TEMs.

In Figure 10, the heat exchanger effectiveness is plotted as a function of the facing area ratio in the range of 0.56 to 1.5 for various thermal heat resistances. The facing area ratio is more critical to the lower value of the thermal resistance in the heat sink. When the facing area ratio and 
the overall thermal resistance of the heat sink are 0.56 and $0.15 \mathrm{~K} / \mathrm{W}$, the heat exchange effectiveness is 0.76 , which is similar to the value of the conventional air-to- air heat exchanger. When the value of the thermal resistance is lower than $0.15 \mathrm{~K} / \mathrm{W}$ and the value of the facing area is more than 0.45 , the heat exchanger effectiveness of the TEHP unit is more than 0.81 .

The design of the thermal resistance and heat distribution of the source of the TEM is the main parameter used to meet the target heat exchange performance of the TEHP unit. The thermal resistance of the heat transfer area between the source to the air in the heat sink should be improved. The structure of the fins can be changed to increase the heat transfer area of the heat sink. For example, corrugated fin heat exchanger types may be used. The heat distribution of the TEM is easily obtained by changing facing area ratio. One of the available options is the use of a heat pipe, which is mainly considered as an assistant heat distributor of the heat exchanger in a local cooling system.

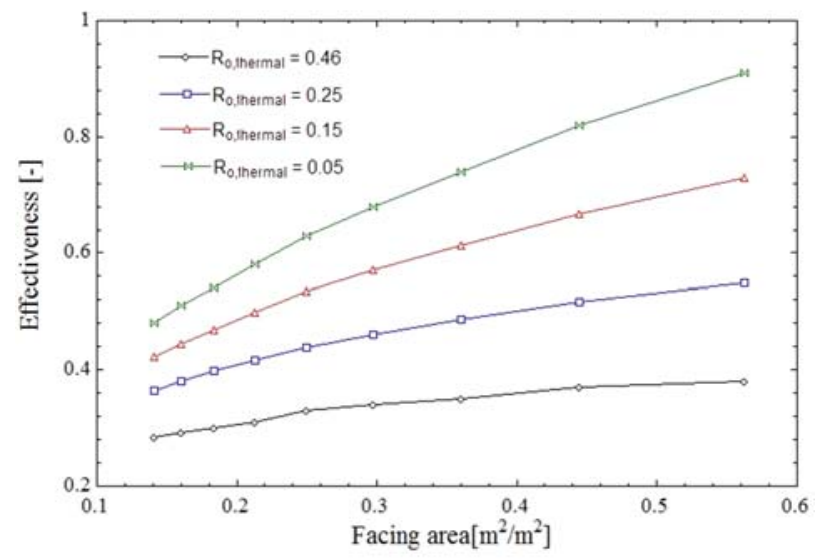

Figure 10: Heat exchange effectiveness as a function of the facing area ratio at various overall thermal resistance values.

\section{Conclusions}

In this study, the main purpose was the proposition of a design for the thermoelectric heat pump unit that could be integrated with a heat exchanger as an air-condenser or evaporator, and the estimation of the heat exchanger effectiveness and outlet air temperature based on numerical analyses. The numerical model was developed for the estimation of a) the effectiveness of the cross-flow heat exchanger type and b) the outlet air temperature. The verification of the numerical simulation was evaluated based on the comparison of actual TEHP unit measurements and simulation data. The simulation values were confirmed based on the estimation of four parameters: cold surface temperature of the TEM, hot surface temperature of the TEM, outlet air temperature of the hot side, and outlet air temperature of the cold side. The maximum difference of the average value to each parameter value was shown to be less than $1.9^{\circ} \mathrm{C}$. While the heat exchange effectiveness of the TEHP unit was developed to generalize the effect of the thermal performance, the accuracy of the estimated value was plotted to vary within $20 \%$ error bounds, based on the comparisons of actual and simulated data. To increase the heat exchange effectiveness, two design parameters were considered: a) the thermal resistance of the heat sink, and b) the facing area ratio of the TEM. The results showed that when the thermal resistance was lower than 0.05 and the value of the facing area was more than 0.45 , the heat exchange effectiveness was higher than 0.81 . To design the TEHP unit, the thermal resistance and the facing area should be considered according to these values by either changing the configuration or by inserting a heat pipe as a heat distributor.

In future research studies, models will be developed for the estimation of the pressure drop and the COP of the TEHP unit according to the heat exchanger configuration.

\section{Acknowledgements}

This work was supported by the Korean Agency for Infrastructure Technology Advancement (KAIA) grants (19CTAP-C141826-02), and by the Korean Institute of Energy Technology Evaluation and Planning (KETEP) (No. 20184010201710).

\section{References}

Creazzo J A, Hammel H S, Cicalo K J, Schindler P (1995). Zero-ODP blowing agents for polyurethane foams. Journal of Cellular Plastics 31, 154-176.

Rowe D M. (2005). Thermoelectrics handbook: macro to nano. CRC Press. Boca Raton (USA).

Lee H S, Attar A M, Weera S L. (2015). Performance prediction of commercial thermoelectric cooler modules using the effective material properties, Journal of Electronic Material 44. 2157-2165.

Lim H S, Jeong, J W. (2018). Energy saving potential of thermoelectric modules integrated into liquid desiccant system for solution heating and cooling. Applied Thermal Engineering 136, 49-62.

Kim M W, Park J Y, Jeong J W. (2017). Energy saving potential of a thermoelectric heat pump-assisted liquid desiccant system in a dedicated outdoor air system. Energies 10, 1306. 